\title{
INTEGRABLE SYSTEMS ON THE SPHERE WITH ELASTIC INTERACTION POTENTIALS
}

\author{
V. V. Kozlov and Yu. N. Fedorov
}

1. The classical example of a system soluble by the method of separation of variables using elliptic spherical coordinates (elliptische Kugel coordinaten) is the Neumann problem on the motion of a point along the $n$-dimensional sphere

$$
S^{n}=\{(x, x)=1\}, \quad x=\left(x_{0}, x_{1} \ldots, x_{n}\right) \in \mathbb{R}^{n+1}
$$

in a force field with the potential energy

$$
V=\frac{1}{2}(A x, x)
$$

where $A: \mathbb{R}^{n+1} \rightarrow \mathbb{R}^{n+1}$ is a symmetric linear operator. The proof of this fact can be found, for example, in [1].

The Neumann system is sometimes called an anisotropic harmonic oscillator on the sphere [2]. This name appears to be related to the fact that if restriction (1) is absent, then the system

$$
\ddot{x}=-\partial V / \partial x
$$

with the positively defined potential (2) can be reduced to a system of disconnected harmonic oscillators. In fact, as we shall see from what follows, an analog of the Hooke potential on the sphere is a function of the form

$$
V(x)=\alpha_{i} / x_{i}^{2}, \quad \alpha_{i}=\text { const }, \quad i=0,1, \ldots, n
$$

To clear up the question we consider the case $n=3$. In [3] the motion of a point along $S^{3}$ in a potential field is studied in the case where the potential depends only on the distance to a center in $S^{3}$. Moreover, the Bertrand problem is solved, which is to find all potentials for which the almost all orbits are closed. As the distance one can take the angular coordinate $\theta$ on a large disk, counted from an attracting (repulsing) center. It turns that, as in the zero curvature space, the Bertrand problem on $S^{3}$ has only two solutions

$$
V=\alpha \operatorname{ctg} \theta, \quad V=\frac{\beta}{2} \operatorname{tg}^{2} \theta ; \quad \alpha, \beta=\text { const }
$$

The first solution is an analog of the Newton potential. As noted in [4], this function satisfies the LaplaceBeltrami equation on $S^{3}$. The second solution is an analog of the elastic spring potential. This function has singularities on the equator $\theta=\pi / 2$. In [4] analogous results are also obtained for the Lobachevskii space $\mathbb{L}^{3}$, and in [5] a detailed analysis of orbits is given (in particular, analogs of the classical Kepler laws for a Newton type potential are deduced). Note that orbits on the $n$-dimensional sphere in a "central" field with potentials (4) are closed for all values $n$; however, a Newton type potential is a harmonic function on $S^{n}$ only for $n=3$.

This work was supported by the Russian Foundation for Fundamental Research, Grant No. 93-013-16244.

Moscow State University. Translated from Matematicheskie Zametki, Vol. 56, No. 3, pp. 74-79, September, 1994. Original article submitted February 18, 1994. 
It is easy to see that if we place the mentioned center of elastic attractio: or repulsion at one of the points

$$
( \pm 1,0, \ldots, 0), \ldots(0, \ldots, 0, \pm 1)
$$

then, up to an insignificant constant, the potential of the force field has the form (3). For $n=2$, the integrability of the system with this potential is established in [4] as a particular case of the integrable generalization of the classical Euler-Lagrange problem for two fixed centers.

By analogy with the potential (2) of a Neumann system, we now consider the motion along $S^{n}$ in the field with the potential

$$
W(x)=\frac{1}{2} \sum_{i=0}^{n} \frac{\alpha_{i}}{x_{i}^{2}}
$$

having $(n+1)$ centers of elastic attraction or repulsion at the points (5). From the Lagrange equations with multiplier $\lambda$

$$
\ddot{x}_{i}=\frac{\alpha_{i}}{x_{i}^{3}}+\lambda x_{i}, \quad i=0,1 \ldots, n
$$

and from (1) we obtain

$$
\lambda=-(\dot{x}, \dot{x})-2 W(x) \text {. }
$$

Thus, unlike the Neumann problem, the multiplier $\lambda$ is a constant, the doubled total system energy with the minus sign, and on $S^{n}$ the system can be reduced to $(n+1)$ disconnected oscillators, i.e., it is trivially integrated in quadratures. In fact, each of Eqs. (6) has the corresponding first integral

$$
\dot{x}_{i}^{2}-\lambda x_{i}^{2}+\alpha_{i} / x_{i}^{2}=h_{i}, \quad h_{i}=\text { const }, \quad h_{0}+h_{1}+\cdots+h_{n}=-2 \lambda ;
$$

whence

$$
\frac{d}{d t}\left(x_{i}^{2}\right)=2 \sqrt{\lambda x_{i}^{4}+h_{i} x_{i}^{2}-\alpha_{i}} .
$$

If both roots $\mu_{1}<\mu_{2}$ of the polynomial $\lambda y^{2}+h_{i} y-\alpha_{i}$ are positive, then the coordinate $x_{i}$ varies between $\sqrt{\mu_{1}}$ and $\sqrt{\mu_{2}}$; if only the root $\mu_{2}$ is positive, then $-\sqrt{\mu_{2}} \leq x_{i} \leq \sqrt{\mu_{2}}$. In the second case, the point on $\mathbb{S}^{n}$ intersects the equator $x_{i}=0$ and the velocity $\dot{x}_{i}$ becomes infinite at the moment of intersection.

If $\alpha_{1} \geq 0, \ldots, \alpha_{n+1} \geq 0$, then solutions of Eqs. (6) are periodic functions, free of singularities, of the same period $2 \pi / \sqrt{-\lambda}$ which depends only on the total energy. Thus, as in the case of one center of elastic interaction, any trajectories of the system under consideration are closed curves. $T S^{n}$,

In the general case, these equations have $n(n+1)$ quadratic integrals on the $2 n$-dimensional phase space

$$
M_{i j}=\left(\dot{x}_{i} x_{j}-\dot{x}_{j} x_{i}\right)^{2}+x_{i} \frac{\alpha_{j}}{x_{j}^{2}}+x_{j} \frac{\alpha_{i}}{x_{i}^{2}}, \quad i, j=0,1, \ldots, n,
$$

and, as in the case of inertia movement, there are $(2 n-1)$ independent integrals.

2. It is known that some classical problems on the motion of a point are also integrable by adding an elastic interaction potential. For example, the Jacobi problem on the free motion of a point along an ellipsoid in $\mathbb{R}^{3}[6]$ remains integrable if the center of elastic attraction (repulsion) is placed at the center of the ellipsoid. Let us show that an analog of this generalized system is also an integrable system on the $n$-dimensional sphere. Namely, assume that a potential field $W(x)$ is given; let us consider the motion of a point along the $(n-1)$-dimensional surface $\mathcal{E}$ that is the intersection of the sphere (1) and a cone

$$
x_{0}^{2} / a_{0}+\cdots+x_{n}^{2} / a_{n}=0, \quad a_{0}<a_{1}<\cdots<a_{n} .
$$

This cone can be included into the family of confocal cones with a parameter $s$,

$$
\frac{x_{0}^{2}}{a_{0}-s}+\cdots+\frac{x_{n}^{2}}{a_{n}-s}=0
$$


It is easy to see that $n$ roots $s=u_{1}, \ldots, u_{n}$ of Eq. (7) correspond to each fixed point on $S^{n}$. These roots are the elliptic spherical coordinates of the point; moreover,

$$
x_{i}^{2}=\frac{\left(a_{i}-u_{1}\right) \cdots\left(a_{i}-u_{n}\right)}{\prod_{j \neq i}\left(a_{i}-a_{j}\right)}, \quad i=0,1, \ldots, n \text {. }
$$

Put $u_{n}=0$. Then the coordinates $u_{1}, \ldots, u_{n-1}$ give the position of the point on the generalized ellipsoid $\mathcal{E} \subset S^{n}$. Taking into account relations (8), the potential $W(x)$ and the kinetic energy $T=(\dot{x}, \dot{x}) / 2$ can be rewritten in these coordinates as

$$
\begin{gathered}
W(x)=\sum_{i=0}^{n} \frac{\alpha_{i} \prod_{j \neq i}\left(a_{i}-a_{j}\right)}{\left(a_{i}-u_{1}\right) \cdots\left(a_{i}-u_{n-1}\right) a_{i}}=\sum_{k=1}^{n-1} \frac{1}{\prod_{l \neq k}^{n-1}\left(u_{k}-u_{l}\right)} \sum_{i=0}^{n} \frac{\beta_{i}}{a_{i}\left(a_{i}-u_{k}\right)}, \quad \beta_{i}=\alpha_{i} \prod_{j \neq i}\left(a_{i}-a_{j}\right), \\
T=\frac{1}{2} \sum_{k=1}^{n-1} \frac{u_{k} \prod_{l \neq k}^{n-1}\left(u_{k}-u_{l}\right)}{\Phi\left(u_{k}\right)} \dot{u}_{k}^{2}, \quad \Phi\left(u_{k}\right)=\left(u_{k}-a_{0}\right)\left(u_{k}-a_{1}\right) \cdots\left(u_{k}-a_{n}\right) .
\end{gathered}
$$

By the Stekkel theorem (see, for example, [7]) the system with the Hamiltonian $T(u, \dot{u})+W(u)$ is integrated in quadratures by separation of variables. In fact, using this theorem, we can show that the system has the following family of quadratic integrals:

$$
I(\varkappa)=\sum_{k=1}^{n-1} \frac{\left(\varkappa-u_{1}\right) \cdots\left(\varkappa-u_{n-1}\right)}{\varkappa-u_{k}}\left(\frac{u_{k} \prod_{l \neq k}^{n-1}\left(u_{k}-u_{l}\right)}{\Phi\left(u_{k}\right)} \dot{u}_{k}^{2}+\frac{1}{\prod_{l \neq k}^{n-1}\left(u_{k}-u_{l}\right)} \sum_{i=0}^{n} \frac{\beta_{i}}{a_{i}\left(a_{i}-u_{k}\right)}\right)
$$

that is a polynomial of power $(n-2)$ in $\varkappa$. Let us fix the constants by putting

$$
I(\varkappa)=\left(\varkappa-c_{1}\right) \cdots\left(\varkappa-c_{n-2}\right), \quad c_{1}, \ldots, c_{n-2}=\text { const } .
$$

From this, equating $\varkappa=u_{k}$, we obtain

$$
\frac{u_{k}\left[\prod_{l \neq k}^{n-1}\left(u_{k}-u_{l}\right)\right]^{2}}{\Phi\left(u_{k}\right)} \dot{u}_{k}^{2}+\sum_{i=0}^{n} \frac{\beta_{i}}{a_{i}\left(a_{i}-u_{k}\right)}=\left(u_{k}-c_{1}\right) \cdots\left(u_{k}-c_{n-2}\right)
$$

or

$$
\dot{u}_{k}=\frac{\sqrt{R\left(u_{k}\right)}}{u_{k} \prod_{l \neq k}^{n-1}\left(u_{k}-u_{l}\right)}, \quad R\left(u_{k}\right)=u_{k} \Phi\left(u_{k}\right)\left[\left(u_{k}-c_{1}\right) \cdots\left(u_{k}-c_{n-2}\right)+\sum_{i=0}^{n} \frac{\beta_{i}}{a_{i}\left(a_{i}-u_{k}\right)}\right] .
$$

It is obvious that $R\left(u_{k}\right)$ is a polynomial of power $2 n$. The expressions we have obtained yield the system of $(n-1)$ equations

$$
\frac{u_{1}^{k} d u_{1}}{\sqrt{R\left(u_{1}\right)}}+\cdots+\frac{u_{n-1}^{k} d u_{n-1}}{\sqrt{R\left(u_{n-1}\right)}}=\varepsilon_{k} d t, \quad k=1, \ldots, n-1
$$

where $\varepsilon_{1}=\cdots=\varepsilon_{n-2}=0, \varepsilon_{n-1}=1$. From this, substituting $d t=u_{1} \cdots u_{n-1} d \tau$, we get the Abel equations for hyperelliptic integrals of the first kind

$$
\begin{gathered}
\int_{u_{0}}^{u_{1}} \frac{u^{k-1} d u}{\sqrt{R(u)}}+\cdots+\int_{u_{0}}^{u_{n-1}} \frac{u^{k-1} d u}{\sqrt{R(u)}}=\delta_{k} \tau+\Delta_{k}, \quad k=1, \ldots, n-1 \\
\delta_{1}=1, \delta_{2}=\cdots=\delta_{n-1}=0, \quad \Delta_{1}, \ldots \Delta_{n-1}, u_{0}=\text { const } .
\end{gathered}
$$


Applying the inversion procedure for Eq. (9) (see, for example, [8]), one cin sxpress symmetric functions of elliptic coordinates and, consequently, the Cartesian coordinates $x_{0}, x_{1}, \ldots, x_{n}$ in the theta-functions of new time $\tau$, associated with the hyperelliptic Riemann surface $w^{2}=R(u)$.

3. Analogous results are also valid for a space of constant negative curvature. We change the sphere (1) to the hyperboloid $L^{n}$,

$$
-x_{0}^{2}+x_{1}^{2}+\cdots+x_{n}^{2}=-1
$$

in the $(n+1)$-dimensional pseudo-Euclidean space with the metric

$$
-d x_{0}^{2}+d x_{1}^{2}+\cdots+d x_{n}^{2}
$$

As the potential energy we again take the function $W(x)$, and, as in the case of the sphere $S^{n}$, each term in $W$ is an analog of the elastic string potential (see [4]).

For the Lobachevskii space $L^{n}$ the Lagrange equations (6) have the form

$$
\ddot{x}_{0}=-\lambda x_{0}+\frac{\alpha_{i}}{x_{0}^{3}}, \quad \ddot{x}_{k}=\lambda x_{k}+\frac{\alpha_{i}}{x_{k}^{3}}, \quad 1 \leq k \leq n
$$

Since the potential energy $W$ is a homogeneous function of $x$ of homogeneity power $(-2)$, we see that the multiplier $\lambda$ is equal to the doubled total energy $T+W=h$ (recall that for the sphere we have $\lambda=-h$ ). Thus, in Eqs. (10) we again obtain $\lambda=$ const. This enables us to integrate (10) in elementary functions. As for the sphere $S^{n}$, these equations admit $(2 n-1)$ independent integrals that are quadratic in velocities.

One can show that the problem of the motion along the $(n-1)$-dimensional "ellipsoid" in $L^{n}$ remains totally integrable by adding a field of elastic forces whose centers are at points chosen similar to. (5). In this case, the separation of variables is carried out in accordance with the scheme discussed in Sec. 2.

\section{REFERENCES}

1. Yu. Mozer, "Some aspects of integrable Hamiltonian systems," Usp. Mat. Nauk, 36, No. 5, $109-151$.

2. A. P. Veselov, "Confocal Surfaces and Integrable Billiards on the Sphere and in the Lobachevskii Space," Preprint. Forschungsinstitute für Mathematik, ETH Zürich.

3. J. Slawianowski, "Bertrand systems on $S O(3, \mathbb{R})$ and $S U(2)$, "Bull. L'Acad. Polonaise Sci., XXVIII, No. 2, 83-94 (1920).

4. V. Kozlov and A. Harin, "Kepler's problem in constant curvature spaces," Celest. Mech. Dynam. Astron., 54, 393-399 (1992).

5. V. V. Kozlov, "On dynamics in spaces of constant curvature," Vestnik Mosk. Univ. Ser. Mat. Mekh. (to appear).

6. K. Jacobi, Lecture in Dynamics [in Russian], Gostekhizdat, Moscow-Leningrad (1936).

7. E. T. Whittaker, Analytical Dynamics [Russian translation], Gostekhizdat, Moscow-Leningrad (1937).

8. B. A. Dubrovin, "Theta-functions and nonlinear equations," Usp. Mat. Nauk, 32, No. 2, 11-80. 